\title{
Unusual tissue distribution of carcinin, an antibacterial crustin, in the crab, Carcinus maenas, reveals its multi-functionality
}

Suzanne Suleiman ${ }^{1}$, Valerie J Smith ${ }^{2 *} \&$ Elisabeth A. Dyrynda ${ }^{1}$

${ }^{1}$ Centre for Marine Biodiversity and Biotechnology, Institute of Life and Earth Sciences, Heriot Watt University, Edinburgh, Scotland EH14 4AS

${ }^{2}$ Scottish Oceans Institute, School of Biology, University of St Andrews, St Andrews, Fife, Scotland KY16 8LB

${ }^{*}$ Corresponding author: vjs1@st-andrews.ac.uk

Key words: WFDSC-domain-containing protein; antimicrobial protein (AMP); haemocytes; ovary; eyestalk peduncle; tissue repair 


\begin{abstract}
Crustins are whey acidic four-disulphide core (WFDSC) domain-containing proteins in decapods that are widely regarded as antimicrobial agents that contribute to host defence. Whilst there have been many analyses of crustin gene expression in tissues, few studies have been made of the distribution of the natural proteins. Here we report an immunostaining investigation of carcinin, a native crustin from Carcinus maenas, in the body organs. The results show that the protein is largely confined to the haemocytes with only a weak signal detected in the heart, hepatopancreas and midgut caecum where it is restricted to the outer surfaces. Importantly, carcinin was seen to be deposited by the haemocytes on these surfaces. Higher levels of staining were detected in the gonads with carcinin particularly abundant in the capsule of ovary as well as some oocytes. Conspicuous staining was further evident in the cuticle of the eyestalk peduncles. Ablation of the eyestalks resulted in a reduction of carcinin in the maturing ovary with the mature eggs rarely displaying a strong signal for the protein. Interestingly, the degree of carcinin also strongly increased in the healing peduncle, indicating that the protein may be associated with wounding, cell damage and/or tissue regeneration.
\end{abstract}

\title{
Highlights
}

- Carcinin protein is mainly present within granulated haemocytes, gonads and eyestalks

- Outer surfaces of other tissues receive carcinin through deposition by haemocytes

- The presence of carcinin in ovaraies changes markedly during oocyte maturation

- Carcinin levels increase significantly in damaged and regenerating tissues 


\section{Introduction}

Antimicrobial proteins (AMPs) occur widely across many animal taxa and are generally considered to play key roles in the innate defences because of their ability to kill or inhibit the growth of bacteria, yeasts and/or fungi. AMPs are particularly important in invertebrates as these animals lack specific antibodies and clonally derived lymphocytes as immune effectors, and so rely heavily on innate immune responses for protection against potential infectious agents. The most studied invertebrate AMPs tend to be those from species of economic or environmental significance, especially decapods and molluscs, as well as antibacterial proteins from species that have value as experimental laboratory models (e.g. insects).

In decapods, one of the most commercially important aquatic invertebrate groups, the dominant taxon-specific AMP families are the crustins and penaeidins. Of these two groups, crustins are the most dominant as they occur widely across the Malacostraca, whereas penaeidins are confined to shrimp and prawn (Smith et al., 2010; Smith and Dyrynda, 2015; Destoumieux-Garzón et al., 2016). In addition to the 50 or so crustins already documented for the Decapoda, crustin-like gene sequences occur more widely across the Pancrustacea, specifically in amphipods, copepods (Smith et al., 2008) and ants (Zhang and Zhu, 2012). Thus these are a noteworthy collection of invertebrate defence proteins and are, accordingly, attracting much research interest.

Crustins are defined as secreted cysteine-rich cationic AMPs of $c$ a 7-14 kDa that have at least one whey acidic four disulphide core (WFDSC) domain at the carboxyl terminus (Smith et al., 2008). Three main types of crustins ((I-III) were originally identified by Smith et al. (2008) based on the number of cys-rich domains (including one WFDSC domain) and the presence, or not, of a glycine-rich domain with 5-8 conserved VGGGLG motifs. However, a fourth type, with a double WFDSC domain, has now also been described (Li et al., 2012) and added to the crustin repertoire.

Certainly, many recent studies of crustins have been directed at mapping the expression of encoding genes in different tissues and quantifying expression changes following various experimental challenges (see review by Smith and Dyrynda, 2015). The reports of these analyses indicate that crustins are mainly expressed in 
haemocytes but appear to be produced also in a variety of other tissues, especially gills, gut, hepatopancreas and haematopoietic tissue (see review by Smith and Dyrynda, 2015). However, whilst gene expression levels in these tissues may change after injection of bacteria, fungi, virus or immune-stimulating compounds, no consistent pattern of response emerges (Smith and Dyrynda, 2015). Clearly what is needed is, first, clarification of the localisation of the mature natural protein(s) in the haemocytes and body organs, and, second, an understanding of where and when crustins are secreted after infection challenge. Such studies are warranted to provide a deeper understanding of where and how proteins exert their effect (Pandey and Mann, 2000). The present study was aimed at addressing the first issue: namely tissue localisation of crustin in vivo.

The crab, Carcinus maenas, was chosen as the experimental animal because its size and tolerance of handing make it a robust and easy decapod to bleed and manipulate. It has few, if any, known lethal pathogens and its robustness has enabled it to spread to many areas of the globe, where it is becoming to be considered as an invasive pest. C. maenas expresses a type I crustin; in fact it was the first WFDSC domaincontaining AMP to be found and purified from any invertebrate (Relf et al., 1999). Cloning and sequencing of this protein, subsequently designated the name, 'carcinin', (Brockton and Smith, 2007) revealed its relationship to glycine-rich WFDSC domain-containing AMPs in other decapod species (Smith et al., 2008). Curiously, carcinin has an unusual pattern of gene expression in vivo following bacterial challenge or temperature change (Brockton and Smith, 2008) but more importantly, in crabs, crustin-type genes are by far the most highly expressed AMP in the haemocytes (Sperstad et al., 2010), making them not only prominent defence molecules but also ones easily and reliably detected at the protein level by immunostaining.

\section{Materials and methods}

\subsection{Animals}

Specimens of adult C. maenas were caught in creels laid in the Forth Estuary, Scotland. Only crabs at moult stage $\mathrm{C}_{4 \mathrm{~T}}$, i.e. those that are fully-grown and past the terminal moult as defined by Drach (1939) and Crothers (1967), were used for 
experiments. These animals ranged in size from $c a 50$ to $75 \mathrm{~mm}$ carapace width. They were maintained in seawater tanks at $c a 10 \pm 3^{\circ} \mathrm{C}$ and $c a 32.5 \pm 1.5^{\circ} \%$ salinity for no longer than 10 days before use. The animals were fed twice per week with commercial fish pellets or fresh mussels.

\subsection{Bleeding and haemocyte separation}

Haemolymph $(2 \mathrm{~mL})$ was extracted from the crabs and diluted in $2.5-3.0 \mathrm{~mL}$ of marine anticoagulant (MAC) as described previously in Söderhäll and Smith (1983). For experiments requiring unseparated haemocytes, the diluted cells were washed in $0.22 \mu \mathrm{m}$ filtered $3.2 \% \mathrm{NaCl}$ and the concentration adjusted to $c a 6 \times 10^{5} \mathrm{~mL}^{-1}$. For experiments on individual populations of each haemocyte type, $2 \mathrm{~mL}$ amounts of freshly drawn diluted haemolymph were loaded onto $9 \mathrm{~mL}$ pre-formed $60 \%$ continuous Percoll gradients made up in $0.22 \mu \mathrm{m}$ filtered $3.2 \% \mathrm{NaCl}$. These were centrifuged at 3,000 $\mathrm{xg}$ for $10 \mathrm{~min}$ to generate bands of hyaline, semi-granular or granular cells (Söderhäll and Smith, 1983). Prohaemocyte populations were obtained and isolated as in Roulston and Smith (2011). Haemocytes, whether used separated or un-separated, were always kept cool on ice and processed immediately. Unless otherwise stated all chemicals and reagents were obtained from Sigma-Aldrich (Irvine, UK).

\subsection{Purification of carcinin}

Haemolymph from 24 adult crabs was extracted as in 2.2 above, except that the MAC used was supplemented with the protease inhibitor, phenylmethanesulfonyl fluoride (PMSF) dissolved in dimethyl sulfoxide (DMSO) to a final concentration of $1 \mathrm{mM}$. The haemolymph samples were pooled and then centrifuged at 1,900 $\mathrm{xg}$ for $10 \min \left(4^{\circ} \mathrm{C}\right)$. After discarding the supernatant, the cell pellet was re-suspended in a volume of ice cold $50 \mathrm{mM}$ sodium phosphate buffer ( $\mathrm{pH}$ 6.5) containing $1 \mathrm{mM}$ PMSF approximately twice the volume of the packed cell volume of the pellet. The haemocytes were then vortexed ( 5 × 2 min bursts) to release the cytoplasmic contents. The haemocytes were cooled on ice for ten minutes between each burst. The disrupted haemocytes were finally centrifuged at $40,000 \mathrm{xg}\left(4^{\circ} \mathrm{C}\right)$ for $22 \mathrm{~min}$. The resulting haemocyte lysate supernatant (HLS) was collected and its protein concentration was determined using the Bradford assay (Bradford, 1976). Typically 
HLS samples derived from 24 crabs contained a total protein concentration of $c a 9$ $10 \mathrm{mg} \mathrm{mL}^{-1}$.

Carcinin was purified from the HLS by liquid protein chromatography on a ÄKTA FPLC system (G.E. Healthcare Life Sciences, Bucks, UK). The procedure was to load $10 \mathrm{~mL}$ of HLS onto a Mono S 5/50 GL cation exchange column (GE Healthcare

Life Sciences) equilibrated with $50 \mathrm{mM}$ sodium phosphate buffer at $\mathrm{pH}$ 6.5. This was eluted with $50 \mathrm{mM}$ sodium phosphate buffer $(\mathrm{pH}$ 6.5) containing $1 \mathrm{M} \mathrm{NaCl}$. The salt gradient was increased from $0-100 \%$ over $20 \mathrm{~min}$. Fractions of $1 \mathrm{~mL}$ were collected and the protein concentration in each was determined by Bradford assay. Fractions containing high levels of protein were subject to a further purification on a Superose 6 10/300 GL gel filtration column (GE Healthcare Life Sciences). Equilibration and elution were again performed using $50 \mathrm{mM}$ sodium phosphate buffer ( $\mathrm{pH}$ 6.5). As the FPLC UV detector revealed single peaks matching carcinin in fractions 19 and 20 on the gel filtration chromatograph, these fractions were collected and subjected to SDS-PAGE and staining with Coomassie Blue R-250 (BioRad, Hertforshire, UK). These produced two close bands of approximately $11 \mathrm{kDa}$ (Supplementary Information Figure 1a), values close to those expected for native carcinin (Relf et al., 1999). Each band was excised and subjected to in-gel digestion (Shevchenko et al., 1996) using a ProGest Investigator digestion robot (Digilab, Champaign, USA) followed by nLC-ESI-MS mass spectrometry, performed by the Mass Spectrometry Unit at the University of St Andrews, which confirmed that both bands were carcinin, presumably as isoforms (Supplementary Information Figures 1b, c).

\subsection{Anti-carcinin antibody production}

Polyclonal rabbit antibody was prepared and purified from $1 \mathrm{mg}$ of purified freezedried pure carcinin by a commercial company (Davids Biotechnologie, Regensburg, Germany) and the resulting product tested for cross reactivity with carcinin in fresh HLS by SDS-PAGE and Western blotting. High resolution SDS page was performed as described by Relf et al. (1999), using a $16 \%$ separating gel, a $10 \%$ spacing gel and a $4 \%$ stacking gel (Schägger and von Jagow (1987). A mini Trans-Blot ${ }^{\circledR}$ Cell (Bio-Rad), with TTBS (Towbin transfer buffer with SDS: $25 \mathrm{mM}$ Tris, $192 \mathrm{mM}$ glycine, 20\% methanol (v/v), 0.025-0.1\% SDS, pH 8.3) was used for blotting on a nitrocellulose membrane at 20 volts for 2.5 hours. The membrane was washed and 
blocked overnight with $3 \%$ bovine serum albumin (BSA) solution in TTBS. Both the anti-carcinin polyclonal antibody and rabbit pre-immune serum were diluted in 3 $\%$ BSA solution in TTBS in different ratios: 1:10,000, 1:50,000 and 1:100,000. Primary incubation was for $1.5 \mathrm{~h}$, followed by three washes in TTBS, each for $5 \mathrm{~min}$. Goat anti-rabbit alkaline phosphatase (AP)-tagged secondary antibody was added at the same dilutions as those listed above, and then incubated for a further $1.5 \mathrm{~h}$. The membranes were again subjected to three washes before addition of alkaline phosphatase substrate solution. This comprised a mix of $100 \mu \mathrm{L}$ of a $15 \mathrm{mg} \mathrm{mL}^{-1}$ solution of 5-bromo-4-chloroindolyl phosphate (BCIP) (made up in dimethylsulfoxide [DMSO]), plus $100 \mu \mathrm{L}$ of nitroblue tetrazolium (NBT) solution (30 mg in $0.7 \mathrm{~mL}$ DMSO and $0.3 \mathrm{~mL}$ distilled water) and $10 \mathrm{~mL}$ of alkaline phosphate colour development buffer (2.5 $\mathrm{mM} \mathrm{MgCl}_{2}$ in $100 \mathrm{mM}$ Tris base $\left.\mathrm{pH} 9.5\right)$. After the signal developed, the nitrocellulose membrane was washed with TTBS and imaged using the ChemiDoc XRS+ system (Bio-Rad). Three controls were included, namely: (i) no primary or secondary antibodies; (ii) secondary antibody only; (iii) pre-immune rabbit serum substituted for the primary antibody. Controls (i) and (ii) used buffer in place of the relevant antibodies. The blots showed that the anticarcinin antibody bound both of the two bands of carcinin (depicted in Supplementary Information Figure 1d) and no other proteins in HLS, thereby demonstrating that it exclusively recognized both isoforms of carcinin.

\subsection{Immunocytochemistry and immunohistochemistry}

The localisation of carcinin in individual haemocyte types and tissue sections taken from at least three healthy crabs was determined by indirect immunostaining.

For analysis of the haemocytes, individual $200 \mu \mathrm{L}$ amounts of separated or unseparated cells, prepared from at least five crabs as described above, were cytocentrifuged on to glass slides for 3 minutes at $250 \mathrm{rpm}$ ( $7 \mathrm{xg}$ ) on a Shandon Cytospin 3 (Thermo-Fisher Scientific, UK) at room temperature. The preparations were then fixed with $4 \%$ paraformaldehyde in $3.2 \% \mathrm{NaCl}$ for $30 \mathrm{~min}$ followed by permeabilization of the cells with Triton-X 100 (3 min) and blocking of endogenous antigens by overnight incubation at room temperature using $10 \%$ goat serum plus 10 $\%$ bovine serum albumin in phosphate buffered saline (PBS) as the blocking reagent. 
The fixed haemocyte preparations were then incubated with the carcinin-specific rabbit antibody (diluted 1:100 in blocking serum) for $90 \mathrm{~min}$. The slides were then re-washed 3 times in PBS (5 min each) and incubated for a further $90 \mathrm{~min}$ in secondary antibody (goat anti-rabbit tagged with FITC: diluted 1 in 100 in blocking solution). Also included with the secondary antibody solution were $5 \mu \mathrm{M}$ Draq 5 (BioStatus Ltd. Leicestershire, UK) (excitation $647 \mathrm{~nm}$; emission $681 \mathrm{~nm}$ ) to reveal DNA and $20 \mu \mathrm{M}$ rhodamine phalloidin (excitation $540 \mathrm{~nm}$; emission $565 \mathrm{~nm}$ ) (Thermo Fisher Scientific, Paisley, UK) to identify actin. Each slide was given another 3 washes of $5 \mathrm{~min}$ in PBS supplemented with $3 \%$ bovine serum albumin before mounting in Vectashield (Vector Laboratories, Peterborough, UK). Control slides comprised preparations without primary or secondary antibodies, samples incubated with secondary antibody only and preparations treated with rabbit sera instead of primary antibody. For each control, $3 \%$ bovine serum albumin in PBS was substituted for the relevant antibody solution. The stained haemocytes were examined by confocal microscopy with a Leica (DMIRE2) TCS2 confocal microscope (Leica Microsystems, Milton Keynes, UK). Images were captured via Leica software and processed using Image J (National Institutes of Health, USA).

To investigate the distribution of carcinin in the main body organs, samples of gill, heart, hepatopancreas and posterior midgut caecum, were excised from three crabs freshly killed by injection of $4 \mathrm{~mL}$ of $2.5 \%$ glutaraldehyde in $3.2 \% \mathrm{NaCl}$ as in Robb et al. (2014). Testes and ovaries were obtained from three male and three female animals respectively. Additional tissues, other than those specified above, were not extracted because the organs were either too small or too fragile to obtain intact. All the excised tissues were immediately fixed in fresh $2.5 \%$ glutaraldehyde for $24 \mathrm{~h}$. The fixed samples were then dehydrated in a graded series of ethanol and cleared with Histo-Clear (National Diagnostics, Yorkshire, UK) in a Shandon Duplex Tissue Processor. The tissue samples were finally embedded in paraffin wax with a Shandon Histocentre 2 Embedding Centre and sections of 4-5 $\mu \mathrm{m}$ thickness were cut before mounting on glass slides and processing for histology and immunostaining. Prior to immunohistochemistry, the sections were rehydrated and permeabilized, as above. Blocking of endogenous antigens was as described above, but with the additional inclusion of $30 \%$ levamisol (Vector Laboratories) diluted with PBS to block any endogenous alkaline phosphatase. The slides were then washed 3 times in PBS (5 
min each) before incubation with the carcinin-specific rabbit antibody at the same dilution and time as for the haemocytes (above). Following a further three washes in PBS, the sections were incubated with the secondary antibody (goat anti-rabbit tagged with alkaline phosphatase) at a dilution of 1:100 in blocking solution. Each slide was re-washed 3 times again and then incubated for $10 \mathrm{~min}$ in the substrate solution, comprising $100 \mu \mathrm{L}$ of the BCIP solution and $100 \mu \mathrm{L}$ NBT added to $10 \mathrm{~mL}$ of development buffer, as in Section 2.4 above. With this substrate solution, carcinin is visualised as a deep blue colouration. After washing the slides in PBS 3 more times the sections were dehydrated through an increasing ethanol series and then finally mounted with Histomount (National Diagnostics, USA). To map organ morphology, additional sections were cut and stained with haematoxylin and eosin to reveal the inner architecture of these tissues. The fine structures on these slides were identified with reference to Johnson (1980). All samples were examined using a Zeiss Axiophot light microscope. Images were captured with ZEN image software.

\subsection{Experimental manipulation of crabs}

To further investigate changes in the presence and distribution of carcinin in the reproductive tissues, eyestalk ablation was performed to provoke gonadal development (Quackenbush, 1986). Eyestalks secrete the neuroendocrine factors that prevent gonad development and moulting, so ablation effectively deprives the animals of these factors. Whilst this has little effect on male crabs, in females it results in an increase in the level of ecdysteroids (moulting hormones) that stimulate ovarian development (Chan, 1995; Subramoniam, 2000). In the present study, the procedure was carried out on both males and females; the males being used as controls for injury and wounding. For both sexes, groups of three crabs were prechilled to $4{ }^{\circ} \mathrm{C}$ to make them quiescent and to reduce blood loss. They were were then subjected to either unilateral (i.e. a single eyestalk) or bilateral (i.e. the two eyestalks) ablation. The procedure entailed swabbing the areas above the $\mathrm{X}$ organs with $70 \%$ ethanol, and then cutting transversally across the middle of the ocular peduncles with sterile scissors. The cut surfaces were immediately treated with antibacterial ointment ( $1 \%$ fusidic acid in a sterile base) after which the animals were allowed to recover for $2 \mathrm{~h}$ at $4{ }^{\circ} \mathrm{C}$ in $0.22 \mu \mathrm{m}$ filtered Instant Ocean (Aquarium Systems Ltd, Cheshire, UK). They were then allowed to return gradually to ambient temperature $\left(10 \pm 3{ }^{\circ} \mathrm{C}\right)$. Non-ablated male and female crabs were similarly 
maintained as controls. Water was changed every $48 \mathrm{~h}$ before the crabs were sacrificed and the gonads were excised and weighed after five days. The gonadosomatic index (GSI) was calculated to estimate gonad maturation for each crab using the following equation:

$$
\text { GSI = gonad weight /body weight } \times 100
$$

After the initial surgery, the excised parts of each eyestalk were fixed in Davidson's solution for $48 \mathrm{~h}$ then immersed in $70 \%$ ethanol before full dehydration and clearing as described in 2.5 above. Glutaraldehyde was not used for fixation because the eyestalks in crabs are partially calcified. Additional peduncle material, ca 2-3 mm thick, was sliced from the top of the severed eyestalks remaining on the crab bodies at 5 days to ascertain if carcinin is associated with wound repair and tissue regeneration of this structure. The slices were fixed and dehydrated as above. All the peduncle samples were then processed for wax histology and immunostaining as described in Section 2.5 above.

\section{Results}

\subsection{Localisation of carcinin in circulating haemocytes in vitro}

Immunostaining with the anti-carcinin antibody showed that freshly harvested unseparated haemocytes give strong signals in the cytoplasm of a proportion (ca $15 \%$ ) of the cells (Figure 1a). The staining was often seen to be so intense as to obscure the cytoplasm and sometimes also the nucleus (Figure 1a). A smaller proportion ( $c a 10$ $\%)$ showed a variable degree of less intense and more granular staining, with the remainder (ca $75 \%$ ) giving no signal at all (Figure 1a). Based on the size, shape and relative proportion in the mix, the cell types would roughly correspond to the granular, semi-granular, and hyaline cells, respectively as described by Smith and Ratcliffe (1978). Within the unstained cell population a few haemocytes were small and had a thin ring of cytoplasm around the central nucleus (Figure 1a), typical of prohaemocytes (Roulston and Smith, 2011).

Further analyses of the four highly enriched haemocyte populations separated on Percoll gradients confirmed that the haemocytes staining most strongly for carcinin were the granular cells (Figure 1b). The extent of co-localisation of the signal with 
rhodamine phalloidin confirmed its intracellular location (Figure 1b). Haemocytes from the semi-granular cell fraction of the Percoll gradients exhibited less and more variable intensity of staining, with the signal largely confined to cytoplasmic inclusions, and rarely showed co-localisation with rhodamine phalloidin (Figure 1c). By comparison, the cytoplasm of haemocytes from the hyaline cell fraction remained unstained for carcinin, although a variable number (up to approximately $30 \%$ ) of these cells displayed a small amount of extracellular carcinin on the outside of the plasma membrane (Figure 1d).

With the prohaemocytes, the enrichment and two-step isolation procedures enabled a greater number of these cells to be examined than would have been possible with freshly drawn haemolymph or single step separation alone, and confocal microscopy established that there are two patterns of staining within this population of haemocytes. The majority were carcinin-negative (Figure 1e) but a few showed some granular staining inside their cytoplasm (Figure 1f).

For all haemocyte types, the pattern of staining was repeated in samples obtained from each of the crabs used.

\subsection{Localisation of carcinin in gills, heart, hepatopancreas, midgut caecum, gonads and eyestalk in vivo}

Immunohistochemical staining of various organ tissues excised from C. maenas consistently showed that a clear signal for carcinin is detectable in circulating haemocytes in situ in all animals used. Figure 2a shows an example of a group of haemocytes residing in the gill. At high magnification the pattern of the distribution of the stain enabled the main haemocyte types to be distinguished consistent with those seen in isolated haemocytes in vitro. Granular and semi-granular cells in particular were discernable by their degree of granular staining with the alkaline phosphatase label. At lower magnification, the gills were seen to be well populated with stained haemocytes (Figure $2 b$ ) but there was no other evidence of carcinin in the lamellae (Figure 2c). Carcinin was also absent from the musculature and internal sub-structures of the heart in each of the three crabs tested (Figure 2d), although weak staining was discernable in the capsules surrounding the outer edges of this 
organ (Figure 2e). Otherwise the haemocytes were the only cells seen to display strong staining with alkaline phosphatase, and these were usually found in the outer regions of the tissue (Figures 2d, e). Analyses of the hepatopancreas similarly revealed that the only cells to show strong staining for carcinin were the haemocytes perfusing the haemal sinuses between the tubules and the associated interstitial connective tissues. Carcinin was absent from the internal matrix of the hepatic lobules, the secretory tubules and the tubule lumens (Figures 3a-d). Instead, it was present only as a thin ring on the outside of the hepatopancreas capsules (Figure 3ad) or as weak patches along parts of the connective tissues on the outer edges of the tubules (Figures 3b-d). Interestingly, in these patches, higher magnification revealed clusters of small dark blue spots in close proximity to stained haemocytes (Figure 3d). These resemble carcinin-positive granules discharged from granulated cells, indicating that carcinin can be deposited on the tissues by exocytosis from granular haemocytes. The distribution of carcinin in the posterior mid-gut caeca followed similar patterns, in that the only positively stained cells were haemocytes present in the interstitial spaces (Figures 3e). There was little evidence of carcinin in association with the caecum basement membranes but carcinin-positive haemocytes were noticed to be in the process of disgorging densely blue material to the extracellular environment (Figure $3 \mathrm{f}-\mathrm{g}$ ). No variations in the staining patterns were observed between the individual crabs.

A different picture emerged for the gonads. In the testes, a positive signal for carcinin was clearly evident not only in haemocytes but also in the connective tissues between the seminiferous lobules and in parts of the epithelia (Figure 4a). It was also evident as a thin layer on the outer edges of the basement membranes but was absent from the lobules themselves, the spermatogonia, the spermatocytes and the spermatids (Figure 4b-d).

Ovaries by contrast, exhibited very strong staining within the ovarian capsules, the thin pavement epithelia of the capsules and some of the developing oocytes, particularly those at the outermost parts of the organs (Figure 5a, c-d). Patches of carcinin were also observed in connective tissues surrounding the developing oocytes and their associated accessory cells within the germinal zone (Figure 5b, c). The signal seen in the epithelia around some of the oocytes in the vicinity of the germinal 
centres may have been deposited by the haemocytes as carcinin-positive cells were commonly observed in very close contact with the epithelia of some oocytes with some appearing to be in the process of depositing stained granular material on to the surfaces of them (Figure 5d). More remarkably, carcinin was further present, to varying degrees, within the cytoplasm of some, but not all, oocytes near to the ovarian walls (Figure 5a). In the more deeply stained oocytes, even the nuclei appeared to contain carcinin (Figure 5a). Again, the distribution of carcinin in testes and ovaries was consistent within the gender groups.

The eyestalk peduncles were the only other body organ in which carcinin was detected at appreciable levels. Samples taken immediately after ablation showed a well-organised architecture comprising an outer acellular cuticle overlying a thin layer of cells, some of which appear to be melanised (Figure 6a). The sub-cuticular endodermal regions beneath this were seen to contain a loose network of interconnected cells, which include neurons (lamina ganglions) and haemocytes amongst others (Figure 6a). Carcinin occurred mainly in the cuticular capsules where it had a distinct pattern of distribution, appearing as patches along the exocuticles and as series of striated bands in the endocuticle (Figure 6b, c). As depicted in Figures $6 \mathrm{~b}, \mathrm{c}$, these endothelial striations show decreasing staining intensity from the outer region inwards, reminiscent of growth rings in tree trunks. The only other staining seen inside the cellular matrices of the inner part of the peduncles was in haemocytes (not shown).

\subsection{Localisation of carcinin following eyestalk ablation}

Macroscopic observations of the ablated C. maenas confirmed the animals were active, feeding and in good condition five days after surgery, so were not seriously handicapped by the treatment. As expected, ablation of the eyestalks had a major impact on the size, texture and colour of the ovaries in female crabs five days after surgery, but had no observable effect on the other body organs or testes in males (Figures $7 \mathrm{a}-\mathrm{c}$ ). In particular the ovaries became more conspicuous and changed from creamy white to yellow and orange in unilaterally and bilaterally ablated animals, respectively, compared to the un-ablated controls. Overall, the effect was much stronger and more pronounced in the individuals in which both eyestalks were removed (Figures 7a-c). These changes were quantified in terms of their GSI values 
as 1.02 for the un-ablated crabs, 1.29 for the unilaterally ablated ones and 4.21 for the bilaterally ablated specimens. These values show that ablation had successfully driven gonadal maturation in the female crabs. As expected, these macroscopic changes were accompanied by marked alterations in the histological characteristics of the ovary tissues, especially a shift in the oocyte profile from the presence of both pre-vitellogenic and vitellogenic stages in the un-ablated crabs to only vitellogenic stages in the treated animals (Figures 7d-f). The unilaterally ablated animals tended to have mostly early vitellogenic oocytes in the ovary (Figure 7e) whereas in the bilaterally ablated females the ovaries were packed with fully mature ova containing yolk bodies (Figure 7f). Regarding carcinin distribution, there was a marked reduction in its level and presence throughout the ovaries in crabs that underwent unilateral eyestalk ablation (Figure 7e). With the exception of a few haemocytes seen in the extra-oocyte vascular spaces, which were well stained, there was very little evidence of staining in the connective tissues between the developing oocytes (Figure 7e). A few oocytes showed weak internal staining but otherwise carcinin was not seen in association with the surrounding epithelia (Figure 7e). Staining was totally absent from the ovaries of the bilaterally ablated animals (Figure 7f) but, more remarkably, these tissues also appeared to be devoid of haemocytes, or at least lacking blood cells displaying positive blue staining (Figure 7f). This could not have been due to haemolymph loss through the surgical procedures as no other organs were similarly affected (data not shown). The only remaining carcinin in these bilateral ablated females was occasionally noted around the ovarian capsules (Figure $7 f)$.

Five days after ablation, however, the eyestalk cuticular capsules seemed to be regenerating despite the structures being ragged and torn with their inner cores unstructured and dense (Figure 6d). Interestingly the regenerating areas were intensely stained for carcinin (Figures 6e-f) with almost the entire exo- and endocuticular areas plus the inner cellular matrices strongly staining blue (Figure 6e). The re-growing inner tissues were also infiltrated with cells, presumably haemocytes, strongly positive for carcinin (Figure 6f). 


\section{Discussion}

There are a growing number of research papers reporting expression of genes encoding immune-relevant proteins in decapod crustaceans with AMPs, including members of the crustin family, popular targets (see review by Smith and Dyrynda, 2015). Many of these studies present data showing that transcripts of crustins are present, to varying degrees, in a wide range of tissues and body organs of unchallenged animals. These investigations include those on crab: Yue et al. (2010) and Mu et al. (2010); shrimp: Sun et al. (2010) and Antony et al. (2011), as well as crayfish (Yu et al., 2016). Such results are often, understandably, interpreted as evidence that the organ or tissue itself expresses the protein. Here we show that carcinin is prominent in the haemocytes, but scarce in several of the body organs in healthy, un-stimulated crabs.

In haemocytes, carcinin is differentially distributed across the various subpopulations, being present only in those haemocytes that contain granules. This distribution of carcinin in the blood cells tallies with the findings of a previous study on the spider crab, Hyas areneus, which determined that the gene encoding a Type I crustin, is expressed over 2,000 times more highly in the granular haemocytes than in the hyaline cells, but only 30 fold more so in the semi-granular cells, again in untreated animals (Sperstad et al., 2010). In the present study we confirm that carcinin in C. maenas is absent from the cytoplasm of the hyaline haemocytes and agranular prohaemocytes, although in vitro it may be external to the plasma membrane of some of these cells. It is likely that the protein was derived from other haemocytes as it is well established that the semi-granular haemocytes of decapods are labile and readily discharge their granules to the exterior in vitro (Bauchau, 1981; Smith and Söderhäll, 1983; Söderhäll et al., 1986). In the present study degranulation could well have been triggered during the bleeding and cell separation procedures. The presence of carcinin in granules of some prohaemocytes is also noteworthy because it provides further evidence that there may be two separate precursor haemocyte lineages in decapods, as previously suggested for shrimp by van de Braak et al. (2002) and subsequently for crab by Roulston and Smith, (2011).

Surprisingly, carcinin was largely absent from gill, hepatopancreas, heart and midgut caecum taken from the un-challenged specimens of $C$. maenas. Where carcinin 
was seen, it tended to be on the outer surfaces of these structures and more conspicuous in haemocytes populating these organs rather than the tissues themselves. As haemocytes were observed disgorging carcinin-positive material at the outer surfaces in contact with the haemolymph, we suggest that the haemocytes are the main source of the carcinin staining apparent in these organs. This raises questions as to the extent to which crustin transcripts, detected in the organs of unchallenged animals by previous authors (cited above) actually originated from tissues themselves or from haemocytes populating them. That there is no consistent pattern of expression levels for individual studies, decapod species, treatments or tissues (reviewed by Smith and Dyrynda, 2015), may be a reflection of this. At present, it is unclear if the distribution of carcinin in the body organs of C. maenas would be different in animals that had received an immune challenge with bacteria, virus or other immune-activating substances compared to untreated ones. So far, our preliminary findings with $C$. maenas (unpublished) lead us to consider that, while the internal body organs receive an influx of haemocytes from the haemolymph following lipopolysaccharide injection, the tissues themselves do not seem to express carcinin de novo as a response.

In contrast to the gill, heart, hepatopancreas and mid-gut caecum, carcinin is present in the eyestalk peduncles. Its occurrence in the endo-cuticle is in keeping with previous studies on crabs that noted the presence of crustin signatures in EST libraries prepared from eyestalks of the crab, Portunus trituberculatus (Liu et al., 2011; Cui et al., 2012). As yet it is unknown whether these transcripts originated from the peduncle tissue itself or from the haemocytes that pervade it. In C. maenas, the striated pattern of staining in the excised peduncles closely resembles that of the growth bands described for the peduncles in other decapod species by Kilada et al. (2012). These bands are believed to form annually in calcified regions of the peduncle in decapods and are often used to estimate the age of the animal (Kilada et $a l ., 2012)$, so it is possible that crustins are synthesised or deposited there by haemocytes as the crab ages and passes through its moult cycles. We propose that the striations of carcinin detected in the peduncles from C. maenas represent individual moult events that occurred during the animals' lives. Mature adult crabs generally moult once per year (Chang, 1995) and during the process the eyestalks, along with the antennae, mouthparts, gills and legs, are withdrawn from the old exoskeleton and 
a new epidermis forms beneath (Crothers, 1967). After the old shell has been cast, the new, soft, epidermis underlying it is finally exposed to the outside environment. Until it hardens, however, the animal is left very vulnerable to injury and infection. It is therefore highly likely that antimicrobial proteins, including crustins, come to be associated, one way or another, with the un-calcified epidermis in order to confer some protection against microbial incursion.

In addition to its location in the eyestalk, carcinin was seen to be conspicuous in the gonads although its presence was more marked in ovaries than in testes. Indeed, in un-ablated crabs both the ovarian tissue itself and some of the developing oocytes near the periphery of the capsules show the strongest staining of all tissues examined except for the haemocytes. Indeed the level is greater than that which would be expected through deposition from haemocytes alone. We therefore believe that synthesis of carcinin can and does occur in the ovary; a view that agrees with the findings of Zhang et al. (2007) and Sun et al. (2010), who reported expression of crustin genes in ovary of untreated shrimp. In crabs, oocytes arise from oogonial cells, and pass through four development stages before maturing into mature eggs (Ravi et al., 2013). These four stages are marked by an increase in cell size with a concomitant decrease in the nuclear:cytoplasmic ratio (Sharifian et al., 2015). There is also a transition from a pre-vitellogenic state to a vitellogenic stage as the oocytes move away from the germinal centre towards the periphery of the ovary (Sharifian et al., 2015). Fully mature eggs are large and packed with yolk bodies (Ravi et al., 2013). In our study, carcinin tended to be located in the larger, vitellogenic oocytes near the outer edges of the tissues. Unfortunately the pre-vitellogenic oocytes in these females were too small to visualise with any degree of certainty but the lack of clear signals in the central region of the ovaries leads us to suppose that they are devoid of carcinin. We do not know why carcinin associates with some of the vitellogenic cells but the heavily vacuolated and fragmented appearance of the carcinin-positive oocytes leads us to suppose that it may play a role in the degeneration, resorption or clearance of defective or redundant ones.

Removal of the eyestalks clearly drove oocyte maturation but, curiously, very little carcinin remained in the fully mature yolk filled eggs of the bilaterally ablated females five days post surgery. Where present it appears to be derived from granular 
material originating from haemocytes. Thus, we conclude that carcinin is confined to oocytes in later vitellogenic development, as it is largely absent from mature eggs.

It is reasonable to assume that the carcinin seen in C. maenas ovary serves in an antimicrobial capacity and therefore should have value in protecting the maturing oocytes from infection. This would be important as mating occurs in crabs when the female has moulted and is susceptible to microbial incursion through the soft, uncalcified epidermis. Certainly, several reports already exist that antimicrobial proteins are associated with the reproductive organs of crabs (Jayasankar and Subramoniam, 1999; Huang et al., 2006; Wang et al., 2007; Qiao et al., 2016; Xu et al., 2011a, b) as well as in some other arthropods (Samakovlis et al., 1991). Indeed there are some WFDSC-domain containing proteins, particularly eppin, that occur in the testes and/or ovaries of mammals (Yenugu et al., 2004; Trexler et al., 2002; see also review by Bingle and Vyacarnam, 2008). However, whilst the notion of gonadal disinfection is attractive and compelling, it does not fit with our observations that carcinin almost entirely disappears from the fully mature eggs and ovaries of ablated female crabs.

The vast majority of decapod crustins, described in the literature are known for their antibacterial activities (Smith et al., 2008; Smith and Dyrynda, 2015) with, as yet, no other functions definitely proven. What is puzzling about these proteins is that their ability to kill or inhibit the growth of bacteria tends to be much weaker than that of other AMP families in the Pancrustacea, and that the proteins mainly act against Gram-positive strains (Smith et al., 2008; Smith and Dyrynda, 2015). In recent years, however, some publications have started to provide indications that, in addition to disinfection, crustins might also have some other physiological effects. For example, a few crustins, primarily Type IIIs (i.e. those that have a relatively simple structure in comprising only one WFDSC domain and a short proline-arginine sequence together with the signal sequence), have proteinase inhibitory properties (Smith et al., 2008). Further, some Type IIs (which possess not only a cysteine rich region but also a long glycine-rich domain adjacent to the signal region) are reported to be involved in haematopoiesis (Fagutao et al., 2012; Chang et al., 2013) or have possible opsonic effects (Liu et al., 2015). Surprisingly, too, expression of a carcinin-like transcript in the swimming crab, Portunus pelagica, has been found be 7-8 times higher in inter- 
moult crabs than at ecdysis, with intermediate levels at pre-and post moult stages (Kuballa and Elizur, 2008). This finding, in particular, is at odds with the notion that the prime role of crustins is in antimicrobial protection because ecdysis is the period when the animal has most need of its host defences.

It is well known that decapod crustaceans are able to regenerate certain parts of their body (especially appendages and eyestalks) after autotomy or injury (Hopkins and Das, 2015). In the present study, the presence of regenerating epithelial tissue seen at the cut surfaces of the peduncles demonstrate that recovery would have been well underway before sacrifice. However, at present it is unclear what purpose carcinin serves in this process. The dramatic increase in the amounts of the protein in the capsular walls at this time point supports the view that carcinin has some involvement in the healing or regeneration processes. Certainly crustins have been reported to be present in regenerating tissues, with PET-15, a Type I crustin transcript in the spiny lobster, Panulirus argus, in particular, expressed at sites of olfactory sensory neuron proliferation (Stoss et al., 2003). Likewise, another Type I crustin gene (designated DW176897) is expressed in regenerating limbs of the fiddler crab, Celuca pugilator (Durica et al., 2006). It is also noteworthy that transcripts of three crustin isoform genes have been identified in the heart, intestine, haemocytes, gills and hepatopancreas of planktonic phyllosoma (larval) stages of Panulirus japonicus, with a fourth isoform detected in nerves (Pisuttharachai et al., 2009). Interestingly, in humans, a gene, encoding a WFDSC-domain containing protein, namely WFDSC-2 (also known as human epididymis protein, HE4), is overexpressed in cancerous ovarian tissue (Hellström et al., 2003). A more recent study has now shown that the protein enhances proliferation of the cancer cells by regulating apoptosis (Chen et al., 2013). Furthermore, in rodents a uromodulin like-1 WFDSC domain-containing protein accelerates age-related ovarian degeneration (Wang et al., 2012). Given that carcinin is not only expressed in regenerating eyestalk, which is well supplied with neural fibres, but is also prominent in ovary and late-stage vitellogenic oocytes, it is not unreasonable to propose that it, and probably other crustins, have some role(s), as yet unknown, in cell and/or tissue repair or regeneration. Further proteomic studies on this family of proteins in other decapods are clearly warranted. 


\section{Acknowledgements}

Funding was provided by a scholarship to SS by the Syrian Ministry of Higher Education, administered by the British Council. The Alumni Fund of Heriot Watt University and the British Council also provided additional financial support to SS. We would like to thank Dr Peter Morris and Prof Stephen Euston (HWU) for advice and other helpful inputs and Mrs Margaret Stobie (HWU) for technical assistance.

\section{References}

Antony, S.P., Singh, I.B., Sudheer, N., Vrinda, S., Priyaja, P., Philip, R., 2011. Molecular characterization of a crustin-like antimicrobial peptide in the giant tiger shrimp, Penaeus monodon, and its expression profile in response to various immunostimulants and challenge with WSSV. Immunobiology 216, 184-194.

Bauchau, A.G., 1981. Crustaceans, in: Ratcliffe, N.A., Rowley, A.F. (Eds.), Invertebrate Blood Cells (Vol. 2). Academic Press, London, pp. 385-420.

Bingle, C.D., Vyakarnam, A., 2008. Novel innate immune functions of the whey acidic protein family. Trends Immunol. 29, 444-453.

Bradford, M.M., 1976. A rapid and sensitive method for the quantitation of microgram quantities of protein utilizing the principle of protein-dye binding. Anal. Biochem. 72, 248-254.

Brockton, V., Hammond, J.A., Smith, V.J., 2007. Gene characterization, isoforms and recombinant expression of carcinin, an antibacterial protein from the shore crab, Carcinus maenas. Mol. Immunol. 44, 943-949.

Brockton, V., Smith, V.J., 2008. Crustin expression following bacterial injection and temperature change in the shore crab, Carcinus maenas. Dev. Comp. Immunol. 32, 1027-1033.

Chan, S., 1995. Possible roles of 20-hydroxyecdysone in the control of ovary maturation in the white shrimp Penaeus vannamei (Crustacea:Decapoda). Comp. Biochem. Physiol. 11, 51-59.

Chang, S., 1995. Physiological and biochemical changes during the molt cycle in decapod crustaceans: an overview, J. Exp. Marine Biol. Ecol. 19, 1-14.

Chang, Y.,T., Lin, C.-Y., Tsai, C.-Y., Siva, V.S., Tsai, H.-J., Song, Y.-L., 2013. The new face of the old molecules: crustin $\mathrm{Pm} 4$ and transglutaminase Type I serving as RNPs down-regulate astakine-mediated hematopoiesis. PLoS One 2793. http://dx.doi.org/10.1371/journal.pone.0072793. 
Chen, Y., Mu, X., Wang, S., Zhao, L., Wu, Y., Li, J., Li, M., 2013. WAP fourdisulfide core domain protein 2 mediates the proliferation of human ovarian cancer cells through the regulation of growth-and apoptosis-associated genes. Oncol. Rep. 29, 288-96.

Crothers, J., 1967. The biology of the shore crab Carcinus maenas (L.) 1. The background-anatomy, growth and life history. Field Stud. 2, 407-434.

Cui, Z., Song, C., Liu, Y., Wang, S., Li, Q., Li, X., 2012. Crustins from eyestalk cDNA library of swimming crab Portunus trituberculatus: molecular characterization, genomic organization and expression analysis. Fish Shellfish Immunol. 33, 937-945.

Destoumieux-Garzón, D., Rosa, R.D., Schmitt, P., Barreto, C., Vidal-Dupiol, J., Mitta, G., Gueguen, Y., Bachère, E., 2016. Antimicrobial peptides in marine invertebrate health and disease. Phil. Trans. R. Soc. B. 371, 20150300.

Drach, P., 1939. Mue et cycle d'intermue chez les crustaces decapodes. Ann. Inst. Oceanogr, Paris, 19, 103-391.

Durica, D.S., Kupfer, D., Najar, F., Lai, H., Tang, Y., Griffin, K., Hopkins, P.M., Roe, B., 2006. EST library sequencing of genes expressed during early limb regeneration in the fiddler crab and transcriptional responses to ecdysteroid exposure in limb bud explants. Integr. Comp. Biol. 46, 948-964.

Fagutao, F.F., Maningas, M.B.B., Kondo, H., Aoki, T., Hirono, I., 2012. Transglutaminase regulates immune-related genes in shrimp. Fish Shellfish Immunol. 32, 711-715.

Hopkins, P.M., Das, S., 2015. Regeneration in crustaceans, in: Chang,E,S, Thiel, M (Eds.), The Natural History of the Crustacea (Vol, 4),. Oxford University Press, Oxford, pp 168-198.

Hellström. I., Raycraft. J., Hayden-Ledbetter. M., Ledbetter. J.A., Schummer. M., McIntosh. M., Drescher. C., Urban. N., Hellström. K.E., 2003. The HE4 (WFDC2) protein is a biomarker for ovarian carcinoma. Cancer Res. 63, 3695-700.

Huang, W.S., Wang, K.J., Yang, M., Cai, J.J., Li, S.J., Wang, G.Z., 2006. Purification and part characterization of a novel antibacterial protein Scygonadin, isolated from the seminal plasma of mud crab, Scylla serrata (Forskål, 1775). J. Exp. Mar. Biol. Ecol. 339, 37-42.

Johnson, P.T., 1980. The histology of the blue crab Callinectes sapidus, a model for the Decapoda. Praeger Publishers, New York.

Kilada, R., Sainte-Marie, B., Rochette, R., Davis, N., Vanier, C., Campana, S., 2012. Direct determination of age in shrimps, crabs, and lobsters. Can. J. Fish. Aquat. Sci. 69, 1728-1733. 
Kuballa, A.V., Elizur, A., 2008. Differential expression profiling of components associated with exoskeletal hardening in crustaceans. BMC Genomics 9, 575.

Jayasankar, V., Subramoniam, T., 1999. Antibacterial activity of seminal plasma of the mud crab Scylla serrata (Forskal). J. Exp. Mar. Biol. Ecol. 236, 253-259.

Li, F., Wang, L., Qiu, L., Zhang, H., Gai, Y., Song, L., 2012. A double WAP domain-containing protein from Chinese mitten crab Eriocheir sinensis with antimicrobial activities against Gram-negative bacteria and yeast. Dev. Comp. Immunol. 36, 183-190.

Liu, Y., Cui, Z., Song, C., Wang, S., Li, Q., 2011. Multiple isoforms of immunerelated genes from hemocytes and eyestalk cDNA libraries of swimming crab Portunus trituberculatus. Fish Shellfish Immunol. 31, 29-42.

Liu, N., Lan, J.F., Sun, J.J., Jia, W.M., Zhao, X.F., Wang, J.X., 2015. A novel crustin from Marsupenaeus japonicus promotes hemocyte phagocytosis. Dev. Comp. Immunol. 49, 313-22.

Mu, C., Zheng, P., Zhao, J., Wang, L., Zhang, H., Qiu, L., Gai, Y., Song, L., 2010. Molecular characterization and expression of a crustin-like gene from Chinese mitten crab, Eriocheir sinensis. Dev. Comp. Immunol. 34, 734-740.

Pandey, A., Mann, M., 2000. Proteomics to study genes and genomes. Nature 405, 837-846.

Pisuttharachai, D., Fagutao, F.F., Yasuike, M., Aono, H., Yano, Y., Murakami, K., Kondo, H., Aoki, T., Hirono, I., 2009. Characterization of crustin antimicrobial proteins from Japanese spiny lobster Panulirus japonicas. Dev. Comp. Immunol. 33, 1049-1054.

Qiao, K., Xu, W.F., Chen, H.Y., Peng, H., Zhang, Y.Q., Huang, W.S., Wang, S.P., An, Z., Shan, Z.G., Chen, F.Y., Wang, K.J., 2016. A new antimicrobial peptide SCY2 identified in Scylla Paramamosain exerting a potential role of reproductive immunity. Fish Shellfish Immunol. 51, 251-62.

Quackenbush, L.S., 1986. Crustacean endocrinology, a review. Can. J. Fish. Aquat. Sci. 43, 2271-2282.

Ravi, R., Manisseri, M.K., Sanil, N.K., 2013. Ovarian maturation and oogenesis in the blue swimmer crab, Portunus pelagicus (Decapoda: Portunidae). Acta Zool. Stockholm 94, 291-299.

Relf, J.M., Chisholm, J.R., Kemp, G.D., Smith, V.J., 1999. Purification and characterization of a cysteine-rich $11.5-\mathrm{kDa}$ antibacterial protein from the granular haemocytes of the shore crab, Carcinus maenas. Eur. J. Biochem. 264, 350-357. 
Robb, C.T., Dyrynda, E.A., Gray, R.D., Rossi, A.G., Smith, V.J., 2014. Invertebrate extracellular phagocyte traps show that chromatin is an ancient defence weapon.

Nat. Commun. 5, 4627.

Roulston, C., Smith, V.J., 2011. Isolation and in vitro characterisation of prohaemocytes from the spider crab, Hyas araneus (L.). Dev. Comp. Immunol. 35, 537-544.

Samakovlis, C., Kylsten, P., Kimbrell, D., Engström, A., Hultmark, D., 1991. The andropin gene and its product, a male-specific antibacterial peptide in Drosophila melanogaster. EMBO J. 10, 163-169.

Schägger, H., Von Jagow, G., 1987. Tricine-sodium dodecyl sulfate-polyacrylamide gel electrophoresis for the separation of proteins in the range from 1 to $100 \mathrm{kDa}$. Anal. Biochem. 166, 368-379.

Sharifian, S., Kamrani, E., Safaie, M., 2015. Oogenesis and ovarian development in the freshwater crab Sodhiana iranica (Decapoda: Gecarcinuaidae) from the south of Iran. Tissue Cell 47, 213-220.

Shevchenko, A., Wilm, M.,Vorm, O., Mann, M., 1996. Mass spectrometric sequencing of proteins from silver-stained polyacrylamide gels. Anal. Chem. 68, 850-858.

Smith, V.J., Fernandes, J.M., Kemp, G.D., Hauton, C., 2008. Crustins: enigmatic WAP domain-containing antibacterial proteins from crustaceans. Dev. Comp. Immunol. 32, 758-772.

Smith, V.J., Dyrynda, E.A., 2015. Antimicrobial proteins: From old proteins, new tricks. Mol. Immunol. 68, 383-398.

Smith, V.J., Ratcliffe, N.A., 1978. Host defence reactions of the shore crab Carcinus maenas (L.): in vitro. J. Mar. Biol. Ass. U.K., 58, 367-397.

Smith,V.J., Roulston, C., Dyrynda, E.A. 2010. The shrimp immune system, in: Alday-Sanz (Ed.), The Shrimp Book. Nottingham University Press, Nottingham, pp. 89-148.

Smith, V.J., Söderhäll, K. 1983. Induction of degranulation and lysis of haemocytes in the freshwater crayfish, Astacus astacus by components of the prophenoloxidase activating system in vitro. Cell Tissue Res. 233, 295-303.

Söderhäll, K., Smith, V.J., 1983. Separation of the haemocyte populations of Carcinus maenas and other marine decapods, and prophenoloxidase distribution. Dev. Comp. Immunol. 7, 229-239.

Söderhäll, K., Smith, V.J., Johansson, M.W., 1986. Exocytosis and uptake of bacteria by isolated haemocyte populations of two crustaceans: evidence for cellular cooperation in the defence reactions of arthropods. Cell Tissue Res. 245, 43-49. 
Sperstad, S.V., Smith, V.J., Stensvåg, K., 2010. Expression of antimicrobial peptides from Hyas araneus haemocytes following bacterial challenge in vitro. Dev. Comp. Immunol. 34, 618-624.

Stoss, T.D., Nickell, M.D., Hardin, D., Derby, C.D., McClintock, T.S., 2004. Inducible transcript expressed by reactive epithelial cells at sites of olfactory sensory neuron proliferation. J. Neurobiol. 58, 355-368.

Subramoniam. T., 2000. Crustaceans ecdysteroids in reproduction and embryogenesis. Comp. Biochem. Physiol. C 125, 135-156.

Sun, C., Du, X.-J., Xu, W.-T., Zhang, H.-W., Zhao, X.-F., Wang, J.-X., 2010. Molecular cloning and characterization of three crustins from the Chinese white shrimp, Fenneropenaeus chinensis. Fish Shellfish Immunol. 28, 517-524.

Trexler, M., Bányai, L., Patthy, L., 2002. Distinct expression pattern of two related human proteins containing multiple types of protease-inhibitory modules. Biol. Chem. 383, 223-228.

van de Braak, C.B.T, Botterblom, M.H.A, Liu, W, Taverne, N.,,van der Knaap, W.P.W., Rombout, J.H.W.M., 2002. The role of the haematopoietic tissue in haemocyte production and maturation in the black tiger shrimp, Penaeus monodon. Fish Shellfish Immunol. 12, 253-272.

Wang, K.-J., Huang, W.-S., Yang, M., Chen, H.-Y., Bo, J., Li, S.-J., Wang, G.-Z., 2007. A male-specific expression gene, encodes a novel anionic antimicrobial peptide, scygonadin, in Scylla serrata. Mol. Immunol. 44, 1961-1968.

Wang, W., Tang, Y., Ni, L., Kim, E., Jongwutiwes, T., Hourvitz, A., Zhang, R., Xiong, H., Liu, H., Rosenwaks, Z. 2012. Overexpression of Uromodulin-like1 accelerates follicle depletion and subsequent ovarian degeneration. Cell Death Dis, 3, e433.

Xu, W.-F., Qiao, K., Huang, S.-P., Peng, H., Huang, W.-S., Chen, B., Chen, F.-Y., Bo, J., Wang, K.-J., 2011. Quantitative gene expression and in situ localization of scygonadin potentially associated with reproductive immunity in tissues of male and female mud crabs, Scylla paramamosain. Fish Shellfish Immunol. 31, 243-251.

Xu, W.-F., Qiao, K., Huang, S.-P., Peng, H., Huang, W.-S., Chen, F.-Y., Zhang, N., Wang, G.-Z., Wang, K.-J., 2011. The expression pattern of scygonadin during the ontogenesis of Scylla paramamosain predicting its potential role in reproductive immunity. Dev. Comp. Immunol. 35, 1078-1090.

Yenugu, S., Richardson, R.T., Sivashanmugam, P., Wang, Z., Michael, G., French, F. S., Hall, S.H., 2004. Antimicrobial activity of human EPPIN, an androgenregulated, sperm-bound protein with a whey acidic protein motif. Biol. Reprod. 71, 1484-1490.

Yu, A.-Q., Shi, Y.-H., Wang, Q., 2016. Characterization of a novel type I crustin involved in antibacterial and antifungal responses in the red claw crayfish, Cherax quadricarinatus. Fish Shellfish Immunol. 48, 30-38. 
Yue, F., Pan, L., Miao, J., Zhang, L., Li, J., 2010. Molecular cloning,

characterization and mRNA expression of two antibacterial peptides: crustin and anti-lipopolysaccharide factor in swimming crab Portunus trituberculatus. Comp. Biochem. Physiol. B 156, 77-85.

Zhang, J., Li, F., Wang, Z., Xiang, J., 2007. Cloning and recombinant expression of a crustin-like gene from Chinese shrimp, Fenneropenaeus chinensis. J. Biotechnol. $127,605-614$.

Zhang, Z., Zhu, S., 2012. Comparative genomics analysis of five families of antimicrobial peptide-like genes in seven ant species. Dev. Comp. Immunol. $38,262-274$. 


\section{Figure legends}

\section{Figure 1. Carcinin distribution in circulating haemocytes}

Cytocentrifuge preparation of haemocytes from C. maenas stained with anti-carcinin antibody. Green staining (FITC) reveals carcinin, blue (Draq 5) reveals DNA and red (rhodamine phalloidin) shows actin. (a) Freshly harvested un-separated haemocytes. Note that carcinin is present only in some cells to varying extent. Many cells remain unstained. $\mathrm{H}=$ hyaline cells; $\mathrm{SG}=$ semi-granular cells; $\mathrm{G}=$ granular cells; PH = prohaemocytes. (b-f) Haemocytes separated on Percoll gradients: (b) Granular cell showing co-localisation of carcinin with actin (yellow staining). (c) Semi-granular cell in which carcinin tends to occur around the nucleus and with less co-localisation with actin than in the granular cells. (d) Hyaline cell with no conspicuous staining for carcinin within the cytoplasm. Peripheral staining outside of the plasma membrane may be from carcinin released from semi-granular or granular cells during cell separation (e) Agranular prohaemocyte lacking carcinin. (f) Granulated prohaemocyte with some carcinin staining evident in the surrounding cytoplasm.

\section{Figure 2. Carcinin distribution in gill and heart}

Paraffin wax sections of gill and heart extracted from healthy adult crabs, stained with anti-carcinin antibody. Carcinin is revealed by alkaline phosphatase label (dark blue). (a) Part of a gill filament showing haemocytes in vivo. The unstained cells are likely to be hyaline cells $(\mathrm{H})$, with patchily stained cells being semi-granular cells (SG) and the more densely stained granular haemocytes (G). (b) Primary gill lamella showing the conspicuous presence of carcinin in the haemocytes (arrow); (c) Secondary lamellae showing carcinin located only in haemocytes (arrows); (d) Cardiac tissue again revealing the absence of carcinin staining except in haemocytes (arrow). (e) Haemocytes staining positively with carcinin clustered at the periphery, whilst the heart tissue itself remains largely unstained apart from weak signals surrounding the outer edge of this organ (arrows).

\section{Figure 3. Carcinin distribution in hepatopancreas and mid-gut caecum}

Paraffin wax sections of hepatopancreas and gut excised from healthy adult crabs and stained with anti-carcinin antibody using alkaline phosphatase (staining dark blue) as label. (a-d) Hepatopancreas. Note the absence of stain in the matrix of the 
organ and the presence of strong staining of the haemocytes perfusing the tissue between the tubules. Weaker staining is visible at the outer edge of the organ in places and in the interstitial connective tissues (arrows). (e-g) Mid gut caecum: The sections are cut across the caecum coils so appears as rings of tissue populated by intensively stained haemocytes (arrows). (e) Staining is largely confined to haemocytes with some densely stained haemocytes appearing to disgorge material (boxed area). (f) Higher magnification of the boxed area from (e) confirming that darkly stained material (arrow) is discharged from carcinin positive cells, likely granular cells. (g). Similar release of intensely stained material (arrow) from a granular haemocyte lying close to the interstitial cells

\section{Figure 4. Carcinin distribution in testes}

Paraffin wax sections of testes extracted from healthy adult male crabs and stained with anti-carcinin antibody using alkaline phosphatase. A positive signal is seen as intense blue. (a-b) Low power plans showing the seminiferous lobules (SL) that do not give a positive signal for carcinin although staining is clearly evident as a thin layer on the outer edge of the basement membrane and in the connective tissue between them (arrows). (c, d) Part of a testis showing spermatagonia, spermatocytes and spermatids (Sp). Note only the haemocytes and patches of the epithelium surrounding the spermatogonia show the blue stain for alkaline phosphatase (unlabelled arrows).

\section{Figure 5. Carcinin distribution in ovary of adult intermolt crabs}

Paraffin wax sections of ovary extracted from healthy adult female crabs. Carcinin is indicated by blue from alkaline phosphatase stain. (a) Low power plan of an ovarian capsule containing oocytes in various stages of development. Note there is very strong staining around the outer edge of the capsule (white arrow), in the thin pavement epithelium of some oocytes and even in some maturing oocytes near the periphery of the capsule (black arrows). There are also a few nuclei showing positive staining for carcinin (grey arrows). (b) Vitellogenic oocytes showing the presence of carcinin in the connective tissue surrounding the developing oocytes. (c) Vitellogenic oocyte with patches of carcinin on its outer surface (black arrow) and an associated accessory cell (white arrow). (d) Higher power detail of a vitellogenic oocyte in very 
close association with haemocytes, some of which appear to be releasing intensely stained carcinin onto the oocyte surface (arrows).

\section{Figure 6. Carcinin distribution in eyestalk peduncle of unablated and ablated female crabs}

Paraffin wax transverse sections of the eyestalk peduncle excised from adult, intermoult female crabs. (a) Appearance of the peduncle in a control slide without carcinin antibody. No blue staining is evident. (b) Appearance of the peduncle at the time of ablation stained for carcinin with an alkaline phosphatase label (blue). Carcinin can be seen in a wide band lying between a thin darkly stained layer of melanin (M) and underneath the outer shell (OS) of the peduncle. (c) Higher power detail of the carcinin-positive region of the peduncle. Note the blue striations of carcinin that diminish in width and colour intensity from the outer region inwards. Each striation represents a previous moult. (d) Haematoxylin and eosin stained section of the regenerating peduncle 5 days after eyestalk ablation. Note the fragmented nature of the damaged tissue. (e) Regenerating eyestalk peduncle 5 days after ablation stained with the alkaline phosphatase label to identify carcinin. The wound is very intensely stained and with a positive signal extending into the inner regenerating tissue (RT) of the structure as well as to the outer capsule. (f). Higher power detail of the regenerating peduncular tissue 5 days post ablation. Note the presence of haemocytes in the regenerating tissue (RT) outside of the internal structure (IS). At least one haemocyte (arrow) seems to be depositing carcinin to the injury area lying between the RT and the IS.

\section{Figure 7. Carcinin distribution in ovary of eyestalk ablated female crabs}

(a-c) Dissected female crabs showing changes in the ovary (arrows) following eyestalk ablation. (a) normal ovary in an un-ablated intermoult female crab: $G=$ gills, $\mathrm{Ht}=$ heart, $\mathrm{Hp}=$ hepatopancreas, the asterix indicates the region where the mid-gut caecum is located. (b) Ovary in a unilaterally-ablated female 5 days post surgery. The tissue is enlarged and developing a yellow colouration. (c) Ovary in a crab subjected to bilateral eyestalk ablation 5 days previously. The ovary is very enlarged and bright orange in colour due to the accumulation of yolk bodies in the oocytes. (d-f) Paraffin wax sections of the ovary tissue stained for carcinin with alkaline phosphate. (d) Ovary from an un-ablated crab showing oocytes at late pre- 
vitellogenic stages and a few early vitellogenic stages of maturation. Carcinin is present in the connective tissue surrounding and within some of the vitellogenic oocytes plus their associated accessory cells. (e) Ovarian tissue from a unilaterally ablated animal. The majority of oocytes are vitellogenic but not fully mature. They show little evidence of carcinin staining. Rather the stain is confined to a few haemocytes present in the interstitial spaces. (f) Ovary from a bilaterally ablated female. Note all the oocytes are now fully mature ova full of yolk bodies. There is little or no evidence of carcinin within the ovarian tissue, although there is staining remaining on the ovarian capsule (arrow). 


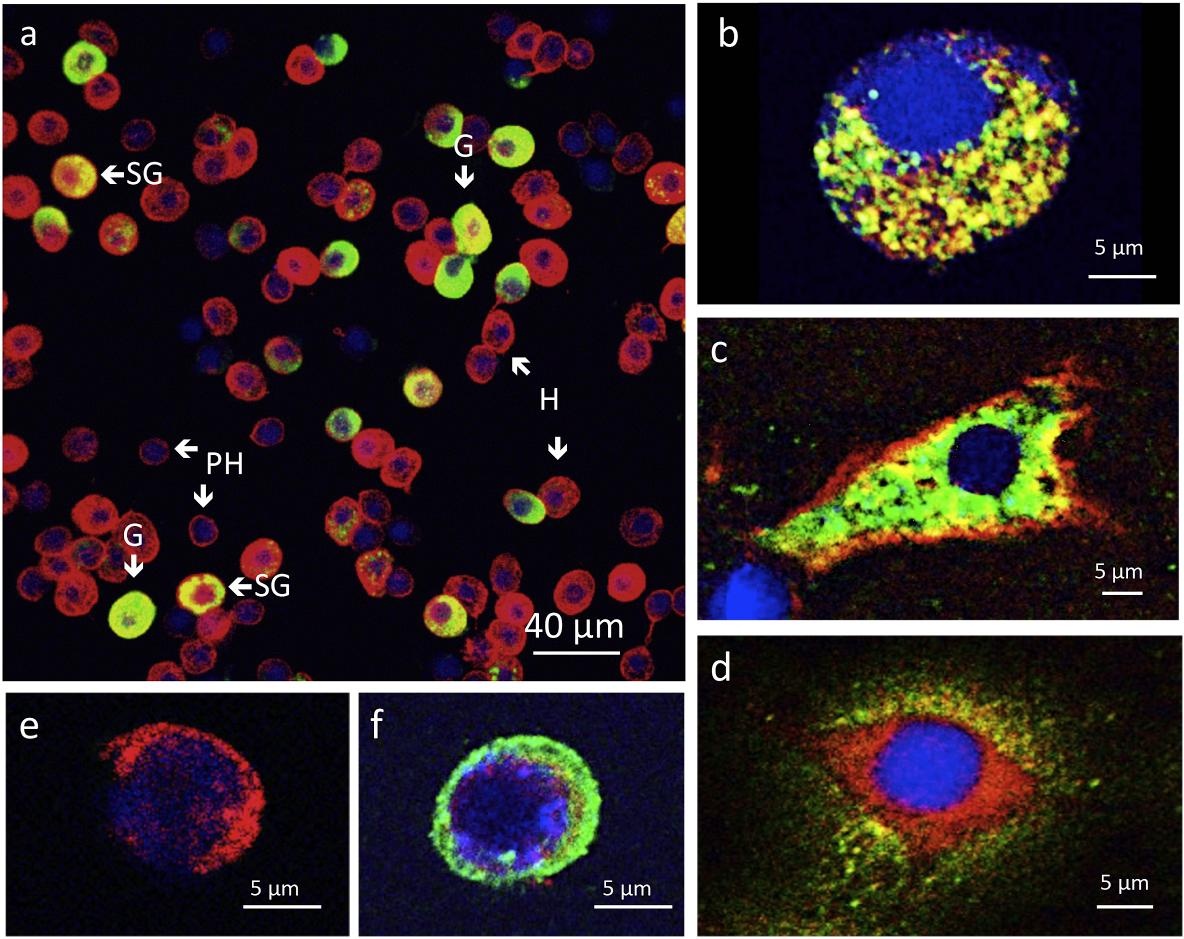

KSG 83 b 


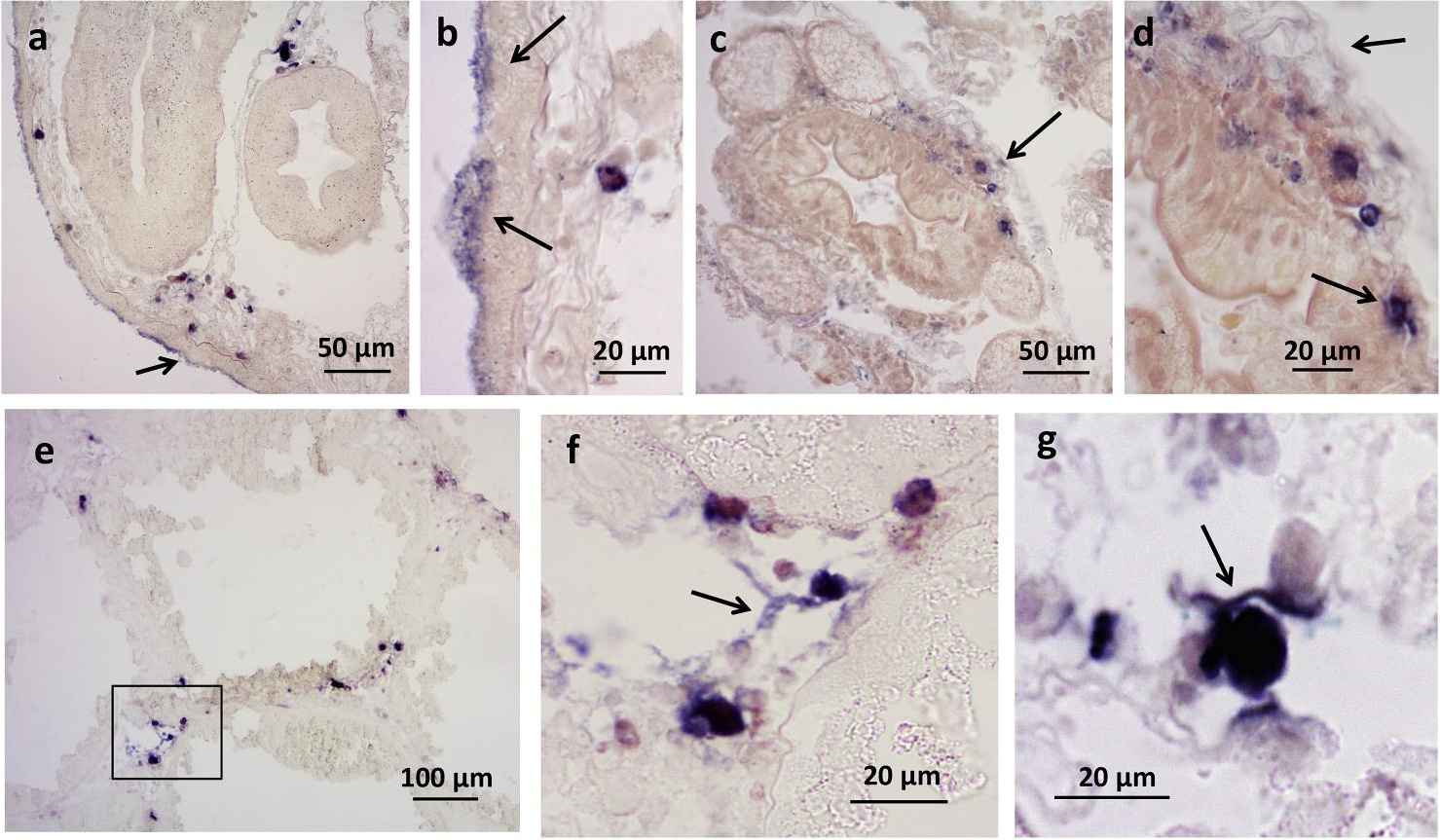




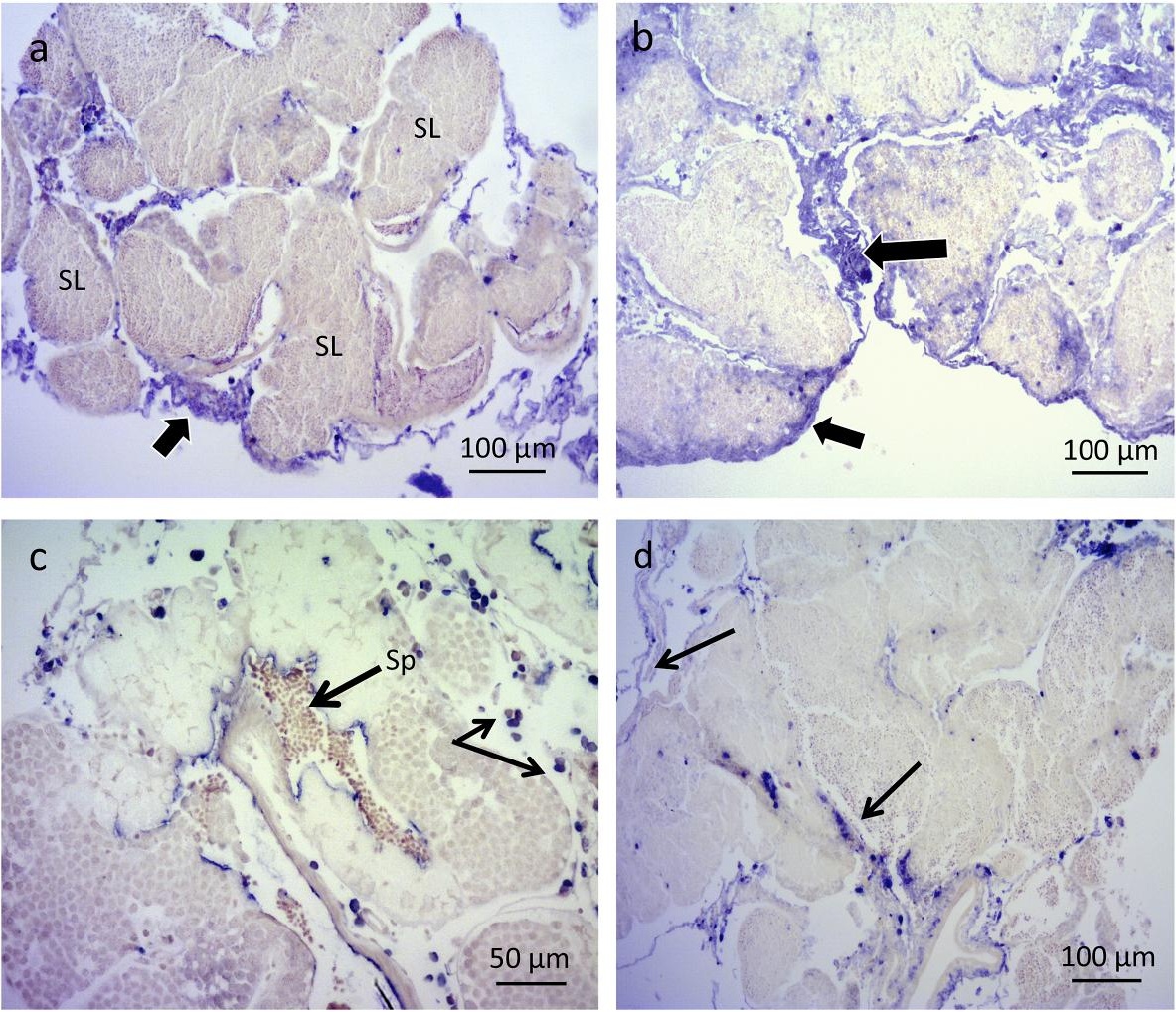




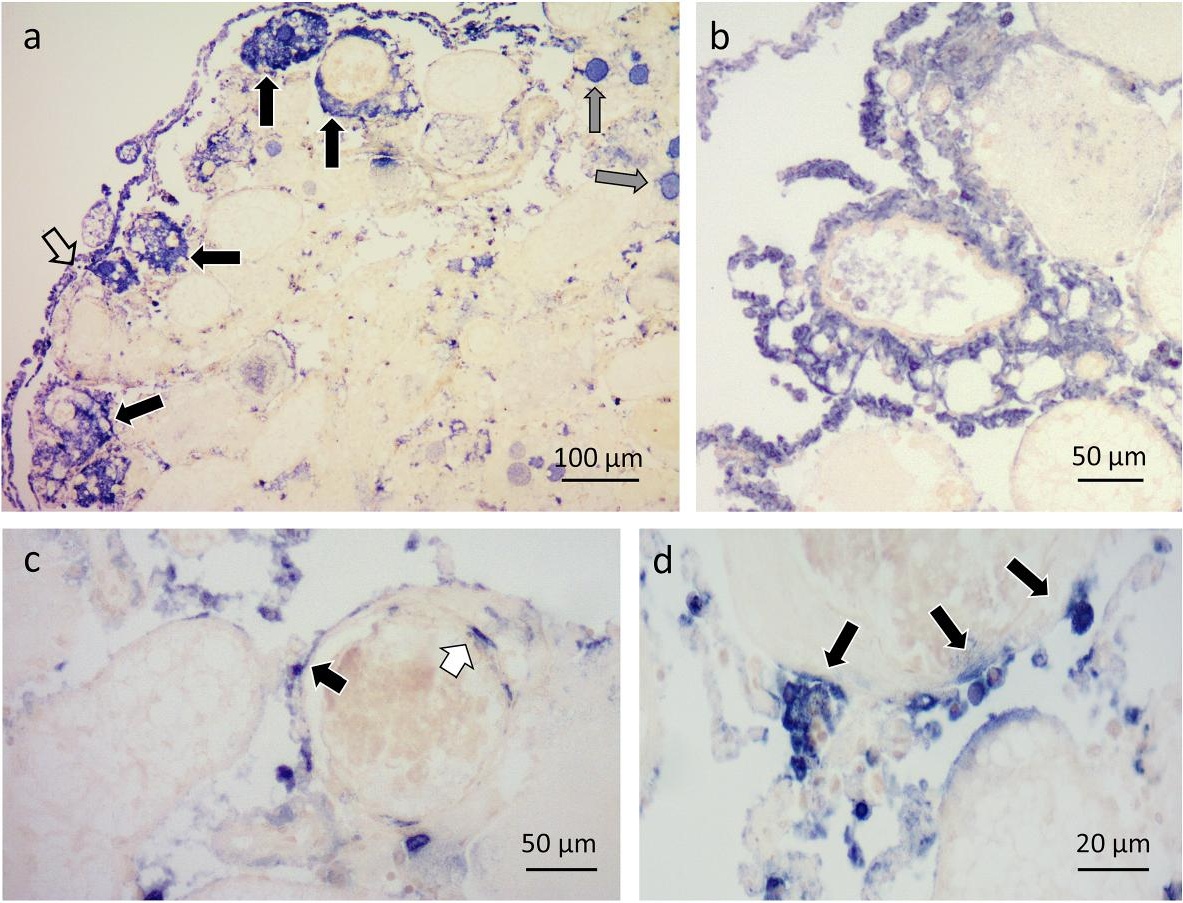




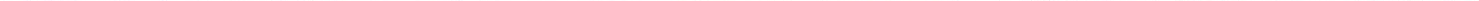

